attributed in each case to iodide released from the contrast medium. Hyperthyroidism is a common disorder and radiological examination of the gall bladder is a frequently performed investigation. Although symptoms were first noted by each patient shortly after cholecystography there is no certainty that their thyroid function was normal beforehand. A causal relationship between the administration of iodide and the development of hyperthyroidism could be inferred if supported by subsequent investigation, by the course of the disorder, and by the response to therapy. The clinical accounts, however, undermine the inference in each case.

In four cases of iodide-induced hyperthyroidism described by Nilsson ${ }^{1}$ the 2-h ${ }^{131}$ I uptake was less than $8 \%$ and in a further four cases which we have investigated the 4-h uptake was below $12 \%$. A 2-h uptake of $52 \%$, as occurred in the first patient described by Drs Fairhurst and Naqvi, suggests that little if any iodide was liberated from the contrast medium and therefore that the onset of hyperthyroidism was coincidental.

The rapid clinical response of both patients to antithyroid drugs is further evidence that their condition was unrelated to their radiographic examination. The hyperthyroidism induced by iodide is sustained by the continuing release of preformed thyroid hormone and not, as is more usual, by the rapid recycling of a limited amount of iodide. In the latter situation antithyroid drugs prevent the recycling process and readily control the condition by blocking the uptake and reuse of iodide released by peripheral deiodination of thyroxine. In iodide-induced hyperthyroidism, in contrast, we have found that the response to drugs is slow; once the serum thyroid hormone concentrations have returned to normal, however, the drugs may be stopped and the patients, unlike Drs Fairhurst and Naqvi's second patient, do not relapse. We would agree with Nilsson that iodide-induced hyperthyroidism, when it occurs in patients who have not had a diet deficient in iodine, is generally a mild, self-limiting condition which requires neither surgery nor treatment with radioiodine.

Although we do not think that the two cases described by Drs Fairhurst and Naqvi are examples of iodide-induced hyperthyroidism, we do believe that this condition is more common in Britain than is supposed. For example, we found ${ }^{2}$ that three out of 29 elderly euthyroid patients given potassium iodide during ${ }^{125}$ I-fibrinogen tests developed biochemical hyperthyroidism. Often in practice the source of the iodide cannot be discovered. The condition can then be surely diagnosed only when hyperthyroid biochemistry is associated with a characteristic mode of handling of radioiodine (a low neck uptake, high urinary excretion, and a low plasma total radioactivity with a large inorganic component).

R L HIMSWORTH MONICA FISHER M J DENHAM

Clinical Research Centre,

Division of Clinical Investigation,

Harrow, Middlesex

1 Nilsson, G, Acta Endocrinologica, 1973, 74, 475 . Aqeing, 1974, 3, 221
Superficial thrombophlebitis

SIR,-The paper by Dr P P Mehta and others (13 September, p 614) shows that heparinoid cream increases the resolution of signs and symptoms of superficial thrombophlebitis developing after continuous intravenous infusion. It also shows that 68 patients had infusions in situ for three days or more. No wonder the authors were able to collect 100 patients with "infusion arms."

Superficial thrombophlebitis developing after intravenous infusion is largely preventable by ensuring that infusions are taken down each night. This is more physiological than running them continuously for 24 hours, relieves night nurses of the particularly difficult task of keeping to infusion schedules in a dark ward, and enables the patients to have a less disturbed night's sleep. Such a policy does require the housemen to insert intravenous cannulae each morning, but as the infusions last no more than 16 hours small needles can be used. These can be put into small veins with relative ease, and blood can be taken through them for analysis before setting up the infusion. In addition it obviates the need to disturb the houseman when an infusion stops in the middle of the night. Since this policy was started on the professorial surgical unit at Guy's three years ago "infusion arms" have been a rare event, and in each case cannulae have been left in the veins for more than 24 hours at a time.

Guy`s Hospital

H G STURZAKER London SEl

\section{An aetiological agent in Whipple's disease?}

SIR,-I read with interest the article by $\mathrm{Dr}$ R L. Clancy and others (6 September, p 568) on the isolation of an aetiological agent from a patient with Whipple's disease. I should like to make two comments.

Firstly, I had difficulty in deciding whether the authors considered the agent to be a cellwall-deficient (CWD) form of Streptococcus dysgalactiae or the bacterium itself. My confusion arose because, on the one hand, jejunal biopsy and lymph node culture materials were considered to contain bacteria as shown by electron microscopy, while on the other hand the authors reported "the isolation of a CWD bacterium from a prolonged lymph node culture with features that strongly suggested that this organism was an aetiological agent." The authors showed the value of using hypertonic medium, but the growth of a CWD form in such medium does not necessarily indicate that it existed in this state in the patient. It cannot be excluded that the streptococcal organisms were in the bacterial form, perhaps in small numbers so that isolation by conventional means was not possible, and that they were converted to the CWD form in vitro by the use of hypertonic medium.

Secondly, even if the isolated streptococcus is the same as that observed in the diseased tissues of the patient, it does not follow that it is necessarily causative. If it is an aetiological agent, then the interesting observations made by the authors are very unlikely to prove unique, even if more than one agent is responsible for causing Whipple's disease. Thus, while in no way decrying the work, I feel that without confirmation it is premature to consider the isolated streptococcus as a cause of the disease on the basis of observations made on a single case.

Clinical Research Centre,

D TAYI.OR-ROBINSON

Harrow, Middlesex

** We showed this letter to Dr Clancy and his colleagues, whose reply is printed below. -ED, $B M$ ' .

SIR,-Two points are made by Dr TaylorRobinson and are well taken.

Firstly, the nature of the isolated organism. The organism was isolated only under cultural conditions that were "characteristic" of those under which cell-wall-deficient (CWD) organisms grow. The initial variation in morphology and staining of the bacteria and the eventual stable growth on blood agar are more consistent with enhanced cell wall stability following isolation than conversion in vitro to CWD forms in the presence of hypertonic medium. The electron micrographic appearance of numerous bacteria in the tissues suggests that the hypertonic medium did not have its effect by allowing growth of organisms present in small numbers. The presence of apparent bacterial cell walls in the electron micrographs neither supports nor excludes functional "cell wall deficiency."

Secondly, is this organism the prime aetiological factor in Whipple's disease? Clearly no definite statement can be made from this study on one patient. Our studies to define the characteristics of this organism, the dramatic clinical improvement following tetracycline therapy associated with a disappearance of bacteria from the tissues, and a reduction in antibody titres to the isolated organism following antibiotic therapy (unpublished) support but do not prove an aetiological role for the organism in our patient. The production of lesions similar to those of Whipple's disease in a rabbit injected with the organism provides further support to this argument.

The questions raised by Dr TaylorRobinson are critical, and for definitive answers further data are required. We certainly do not intend to convey the impression that the organism isolated in our study is necessarily the cause of Whipple's disease but hope we can stimulate study of more patients with this interesting and complex disorder, to understand better its aetiology.

\section{R L CI.ANCY}

W A F TOMPKINS

H RICHARDSON

T J MUCKLE W E RAWLS

Department of Medicine,

Mamaster University.

\section{Diagnostic criteria in the ${ }^{125}$ I-fibrinogen test}

SIR,-Mr V C Roberts's paper (23 August, p 455 ) is a timely and valuable statement of problems encountered with the Pitman ratemeter and we strongly support his plea for standardisation of diagnostic criteria in the ${ }^{125}$ I-fibrinogen test. The need for standardisation is clearly illustrated by our figures. A total of 314 patients were scanned 
from the preoperative day up to the seventh postoperative day with a Pitman ratemeter No 235. Readings as a percentage of heart count were recorded direct. Each patient's scans were analysed according to four different criteria (see below), all of which have been regarded as diagnostic by different authors. The last of these criteria has usually been used in combination with other criteria, and others have used a $15 \%$ differential. ${ }^{2}$ In each instance the scan was regarded as positive only if the criterion was met for 24 hours or more. Confirmation of a diagnostic count has not always formed part of the criteria; not to have done so here would have resulted in a higher number of positives, particularly when analysed by the first criterion, as shown.

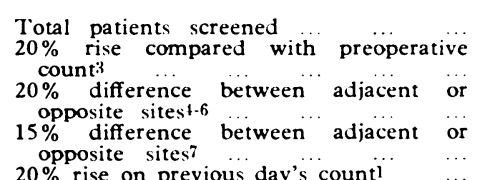

314

73

30

42

Much the highest number of positives is recorded when comparison is made with the preoperative count. Mr Roberts's suggestions for improved accuracy are desirable, but some of the attractions of the ratemeter compared with the scaler are then lost. Whether or not all these suggestions are followed, we think that only criteria which involve comparison on the same day should be used; in this way the effecr of background, which forms an increasing proportion of the count, is minimised and the importance of daily variation in the magnitude of counts reduced. During the first few days, distribution of ${ }^{125}$ I-fibrinogen between intravascular and extravascular spaces is changing and so is the geometry of counting - not only of the legs but of the precordium. We have found a general increase in later leg counts and think that the relative proportion of count formed by extravascular fibrinogen increases more in the legs than the precordium. We realise that the same percentage reading on one day may still represent a different magnitude of counts on another, but we think this is a further reason why comparison with a former count is best avoided. Although the risk of missing bilateral thromboses would seem to be greater if comparison with a former count is excluded, the diagnosis based on suoh criteria correlates well with phlebography. ${ }^{7}$

P ATKINS

I KEITH BROWN G J SANTER Walton Hospital,
Liverpool

P M ROBB

1 Bonnar J, and Walsh, J, Lancet, 1972, 1, 614.

1 Bonnar J, and Walsh, J, Lancet, 1972, 1,

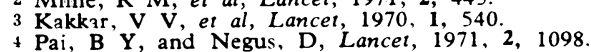
5 Gordon-Smith, I C, et al, Lancet, 1972, 1, 1133. 6 Focsard, D P, et al, Lancet, 1974, 1, 9.

Negus D, et al, British fournal of Surgery, 1968 55, 835

\section{Promiscuity and infertility}

SIR,-With reference to your leading article (30 August, p 501) there may be other effects of promiscuity than that of venereal disease and subsequent infertility. Green-Armytage reported in 1943 from the West London Hospital that a group of 20 recently married women who did not use artificial contraception were observed over a period of two years and compared with a group of similar women for a similar period who used barrier methods. The 20 who did not use artifical contraception developed what GreenArmytage called full maturation of the uterus and a high proportion became pregnant, while the 20 who used barrier methods retained what he called a hypoplastic or premarital state of the uterus and five developed cervical erosions. I have looked through the Index Medicus and made other inquiries but have not been able to find reports of a confirmatory or conflicting nature. Would it not be possible to repeat the investigation using, at the end of two years for two such groups, assays of oestriol levels in the plasma and of macrophage and lymphocyte competence? The interpretation of such assays might be of interest.

Buckfastleigh, Devon

MichaEL WILKINSON 1 Green-Armytage, V B, Proceedings of the Royal
Society of Medicine, 1943, 35, 105.

\section{Complications of central venous}

catheterisation

SIR,-The complications of central venous catheterisation are important, and Dr M J Greenall and his colleagues (14 June, p. 595) have raised some provocative technical points.

Radiographic visualisation of the catheter is essential as soon as practicable after insertion. Only radio-opaque carheters are acceptable. Nevertheless it is not possible always to $x$-ray a patient immediately after insertion of a central venous catheter and before its use for fluid infusion. The following technical points help to reduce the possibility of acute complications.

(1) Advancement of a correctly placed catheter is gentle and easy.

(2) Do not coil a long catheter up in the right atrium (or right ventricle!) by advancing it too far. Location of the tip in the ipsilateral innominate vein is preferable.

(3) After insertion and before fixing the catheter attach a syringe containing isotonic saline, inject a millilitre or so to clear any clot, and aspirate blood into the syringe. Do not use undue force when aspirating. If the tip is lodged incorrectly or against the intima of a central vein excessive force is pointless and perhaps even harmful. Should gentle aspiration not produce free-flowing blood inject a little more saline, withdraw the catheter $2 \mathrm{~cm}$, and repeat the aspiration test. Failure to obtain blood is a contraindication to further use of this catheter.

(4) Connect the intravenous drip and observe that it flows freely. The meniscus in the central venous pressure manometer tubing should fall freely to a "sensible" pressure. Erratic pressure measurements may be an early sign of an incorrectly placed catheter ${ }^{1}$.

(5) Compress each side of the neck separately. A rise in the central venous pressure greater than $10 \mathrm{~cm} \mathrm{H}_{2} \mathrm{O}$ suggests the catheter tip is in the ipsilateral internal jugular vein"2.

(6) Look at the meniscus and look for two distinct oscillatory patterns: (a) a larger amplitude respiratory oscillation, and (b) a smaller amplitude, higher frequency cardiac oscillation. If these are not both visible, do not infuse fluid nor take pressure readings until the aspiration test is successfully repeated.

A posteroanterior chest radiograph as suggested by $\mathrm{Dr}$ Greenall and his colleagues may be impracticable. Will the projection on an anteroposterior chest film serve the same purpose? It is also impracticable that the arm should not be abducted beyond 90 in some patients requiring intensive nursing care and in certain postural requirements in the operating theatre. In infants wide arm abduction is commonplace.

Cannulation of the jugular $r^{3}$ or subclavian ${ }^{4}$ (or, less commonly, femoral) vein avoids a problem from shoulder movement, and, despite the risks ${ }^{67}$ and reasoned arguments for the use of the arm veins, ${ }^{8}$ other veins continue to serve a valuable purpose in experienced and careful hands.

The advice to use only electrolyte solution in the first 12 hours will not necessarily avoid serious consequences of aocidental extravasation. On two occasions in my experience resuscitation using colloid infusion through an urgently cannulated subclavian vein was life-saving by the only adequate venous routc obtainable.

One must question the wisdom of advising "routine" needle aspiration of the pericardial sac in a resuscitation programme for cardiac arrest in a patient with a central venous catheter in situ. A clinician experienced in this technique is rarely likely to be on the scene.

Withdrawal of an existing central venous catheter by $15 \mathrm{~cm}$ before its use in resuscita tion can apply only to a arm (or leg) cathetes if it is not to be pulled out altogether at an inconvenient moment for the patient.

This paper by $\mathrm{Dr}$ Greenall and his colleagues reminds us of two important but not widely appreciated facts. Firstly, cardiac. tamponade and other major perforation com plications can occur during the first minutes following the insertion of a central venous catheter by whatever route. Secondly, a high quality chest radiograph showing the catheter tip correctly and apparently safely placed, although essential, does not exclude the danger of a complication, immediate of delayed.

JOHN WILLIAMSON

Department of Anaesthetics,

Univer

1 Adair, R, and Moses, M, Fournal of the American Medical Association, 1970, 214, 372

Intey, J, and Russell, Intensive Care, 1975, 3, 101.

English I S W, et al, Anaes hesia, 1969, 24, 521.

Yofa. D, Lancet, 1965, 2, 614

Davidson, J T, et al, Lancet, 1963, 2, 1139.
Defalaue, R J, Anaesihesia and Analgesia, 1968, 47. 677 .

Arnold, S, et al, British Medical fournal, 1973, 1,211

Shang Ng. W, and Rosen, M, British fournal of Anaesthesia, 1973, 45, 1211 .

Vaginal cytological examination in anorexia nervosa

SIR,-In connection with the interesting paper by Drs S J Nillius and L Wide (16 August, $p$ 405) I should like to mention a very simple method of monitoring the effect of human chorionic gonadotrophin therapy in amenorrhoeic patients with anorexia nervosa -examination of the vaginal smear. It is easy to perform, quick, and completely harmless. The use of haematoxylin-Shorr staining is advisable.

Of five patients with anorexia nervosa examined before treatment the vaginal smear showed total hormonal ovarian insufficiency in four, while in the other five some oestrogenic effect was demonstrable. There was a 